\title{
A Research on Construction of SOAs for Enterprises in China
}

\author{
Xin Liu, Ming Zha, Tingjie Lu and Wei Fan
}

\begin{abstract}
School of Economics and Management, Beijing University of Posts and Telecommunications, Beijing 100876, P.R. China liuxin1919@gmail.com chaming123456@yahoo.com lutingiie@263.net anwei@buptinfo.com
\end{abstract}

\begin{abstract}
In mainland China, many enterprises still use early client/server technologies for their supply chain management (SCM). However, with China's participating in WHO and economy globalization improving, Enterprises in mainland China have to compete with multinational companies. They have to move from client/server technologies to service oriented architectures (SOA) in order to their SCM can catch up with their international rivals. Many enterprises in mainland China do not have enough money to invest their supply chain systems. In this paper, we propose to investigate the feasibility of using SOA in the construction of innovative and SCM of individual enterprise with numbered money. It starts with an introduction of SOA. A SOA is a form of distributed systems architecture that is typically characterized by the following properties: logical view, message orientation, description orientation, network orientation and platform neutral. The paper highlights the description problem of SOA within the context of the SCM and proposes a modeling framework as solution. It can help enterprises in mainland China to resolve their SCM with numbered money.
\end{abstract}

Keywords: Service management, Service-oriented architecture (SOA), Supply chain management (SCM), Supply chain, Electronic business (E-business)

\section{INTRODUCTION}

Client/server technologies, the IT topics of the 1980 s and 1990 s, have followed a familiar path for technology: from hype, to disillusionment, to maturity, to decline. Early client/server rollouts were costly and unreliable, but now standards and tools evolved, and so did skills and companies begin to reap the advantages of client/server scalability, flexibility and ease of application development.

Since come in 21 st century, developers have discovered that still more tiers can bring better performance, flexibility and scalability. Applications can be broken into presentation, business logic, data access and data storage layers, each residing where it works best. Stir in Web-based clients and Web services, and those advantages are magnified. Apparently, client/server was just a stepping stone. The focus is now on SOA to take up the baton and help us build a new generation of process automation applications that extend the same stream of business information. Using the metaphor

Please use the following format when citing this chapter:

Liu, X., Zha, M., Lu, T., Fan, W., 2007, in IFIP International Federation for Information Processing, Volume 255, Research and Practical Issues of Enterprise Information Systems II Volume 2, eds. L. Xu, Tjoa A., Chaudhry S. (Boston: Springer), pp. 1047-1056. 
of providing a service, the opportunity exists to create solutions to handle a full business request, such as processing an order, a loan, or a claim.

These high-level services are themselves supported by one or more layers of lower level services which perform data lookups, validations, transformations, updates, or other processing tasks previously done manually. Enabled by new programming tools, protocols, and standards for interoperability, services can be invoked effectively between applications on different platforms, in different locations, and using systems owned by different organizations.

Thus, SOA has been paid an increasing attention in both academic and industrial communities since software systems based-on it adapt well to the continuous changes of requirements and application environments.

In China mainland, many enterprises still use early client/server technologies for their SCM. However, with Chinese entry WHO and economy globalization improving, Enterprises in China have to compete with multinational companies. They have to move from client/server technologies to SOA in order to their SCM can catch up with their international rivals'. But, more than 90 percent enterprises in China do not have enough money to invest their service systems. In the paper, it starts with a short introduction of the SOA and then gives a description of SCM. In giving modeling of sharing information in supply chains, the proposed model is a discrete dynamic model and the cooperation of units is based on contracts and formal agreements achieved in negotiation process; in modeling of sharing information in industry chain, the proposed model is a discrete dynamic model and the cooperation of units is based on protocols and formal agreements achieved in negotiation process; In giving a model of sharing services, the proposed model is a sharing service can be represented by four components; at last, it highlights the describing problem of SOA within the context of the SCM and proposes a modeling framework as solution, it help enterprises in China mainland to resolve their SCM with numbered money.

\section{SERVICE-ORIENTED ARCHITECTURE}

SOA is a term that many different people use in many different ways. Most of the time, SOA refers to architecture for software systems in which services are the fundamental building blocks; This is a good, broad definition, but there are many shades of meaning when you start digging into the details of what someone means when they use the term.

Marks, Eric A. (2004) describe SOA's advantages [1]:

The SOA's key advantage is flexibility. Unlike previous paradigms such as clientserver and mainframe environments, it offers IT functionality as cross-platform shared services.

Once a portfolio of Web services is available in an SOA, these reuse benefits multiply exponentially in an "SOA network effect." The value of SOA increases with the number of available services, and the number of different applications or users that access those services. This value compounds over time and becomes even greater if SOA is leveraged both internally and externally. 
An enterprise could start out with an SOA used for internal services, for example, and then expand it to customer-facing applications. This entails XML data exchange with trading partners, a practice that's becoming more common in the telecommunications and travel industries. An SOA can also benefit customer-facing $\mathrm{B} 2 \mathrm{~B}$ applications, where the user is unaware of the underlying infrastructure.

SOA's flexibility also benefits an organization through faster application development and lowered costs by allowing hardware and software components to be reused.

Applications developed this way can even be of higher quality than those developed independently because the components are retested and the Web services interfaces have already been proven.

The speed, ease, and quality of SOA developed applications have led many analysts to see the architecture as a means of aligning an enterprise's goals with its IT services and capabilities. If implemented properly, its flexibility will filter through to the entire network and to the organization as a whole: An IT department can modify technology without making changes to the services available, while an enterprise can change its applications or business processes with minimal effect on the IT architecture.

SOA's flexibility is particularly important when trying to integrate several different systems, such as those that result from mergers. After organic growth and multiple acquisitions, for example, office supply retailer Staples found itself with five duplicate systems for credit card authorizations. Rather than transition to just one authorization system or create multiple interfaces to each back-end application, Staples implemented a Web service for use by all five. An SOA provides the runtime, management, and security functionality, allowing Staples to route each of its 100 million annual credit card transactions through whichever one of the five banks and clearinghouses provides the best value for that particular payment.

SOA refers to any system that exposes its functionality as services. The next question, naturally, is "what's a service?" We will turn to a metaphor to explain that one: think of services as a mechanical watch with hands, numbers, and an internal mechanism. The hands and numbers are the "interface," and the mechanism is the "code." To do more than simply tell time - to function as a stopwatch, for examplea watch would need additional components, such as mechanisms to start and stop the time, to display the elapsed time, and to reset the timer. Those operations are essentially simple services.

To be useful, the service orientation needs to exist everywhere across applications and systems. The emphasis here should be on the word across. It's critical that the service orientation be able to draw upon any application and any database in the system, or else the usefulness of the architecture is crippled from the very beginning.

SOA has led to the creation of many related terms, some that were created because of SOA and others that were given new life. The term loosely coupled, for example, refers to a property of systems in which the complexity of the system is partitioned inside a small number of building blocks that are connected in clearly defined ways. A service grid is an infrastructure of many different services all designed to work together. Many terms such as these are being created every year as new ideas emerge. The World Wide Web consortium [2] defines as follows: A Service Oriented 
Architecture (SOA) is a form of distributed systems architecture that is typically characterized by the following properties:

Logical view: The service is an abstracted, logical view of actual programs, databases, and business.

Message orientation: The service is formally defined in terms of the messages exchanged between provider users and requester users, and not the properties of the users themselves. The internal structure of a user, including features such as its implementation language, process structure and even database structure, are deliberately abstracted away in the SOA: using the SOA discipline one does not and should not need to know how a user implementing a service is constructed.

Description orientation: A service is described by machine process able meta-data. The description supports the public nature of the SOA: only those details that are exposed to the public and important for the use of the service should be included in the description.

Granularity: Services tend to use a small number of operations with relatively large and complex messages.

Network orientation: Services tend to be oriented toward use over a network, though this is not an absolute requirement.

Platform neutral: Messages are sent in a platform neutral, standardized format delivered through the interfaces.

\section{SUPPLY CHAIN MANAGEMENT}

SCM has generated a substantial amount of interest both among managers and researchers. SCM is now seen as a governing element in strategy and as an effective way of creating value for customers. SCM benefits from a variety of concepts that were developed in several different disciplines as marketing, information systems, economics, system dynamics, logistics, operations management, and operations research. There are many concepts and strategies applied in designing and managing supply chains. The expanding importance of supply chain integration presents a challenge to research to focus more attention on supply chain modeling. A structure of supply chains is composed from potential suppliers, producers, distributors, retailers and customers etc. The units are interconnected by material, financial, information and decisional flows. Most supply chains are composed of independent units with individual preferences. Each unit will attempt to optimize his own preference. Behavior that is locally efficient can be inefficient from a global point of view. In supply chain behavior is much inefficiency. An increasing number of companies in the world subscribe to the idea that developing long-term coordination and cooperation can significantly improve the efficiency of supply chains and provide a way to ensure competitive advantage.

The overall business environment is becoming increasingly dynamic. Demand and supply for custom products can be very dynamic. Supply chains operate in a network environment. Dynamic information and decision-making models are called to accommodate these changes and uncertainties. 
There are some approaches to model and analyze the supply chain dynamics. Dynamic models of supply chains try to reflect changes in real or simulated time and take into account that the network model components are constantly evolving. An information asymmetry is a source of inefficiency in supply. Information exchange is a very important issue for coordinating actions of units. The expected result is a mutually beneficial, win-win partnership that creates a synergistic supply chain in which the entire chain is more effective than the sum of its individual parts. Supply chain partnership leads to increased information flows, reduced uncertainty, and a more profitable supply chain. The ultimate customer will receive a higher quality, cost-effective product in a shorter amount of time.

\section{MODELING OF SHARING INFORMATION IN SUPPLY CHAINS}

The layers of producers and retailers compete in a non-cooperative way, but the partners in individual supply chains can profit from cooperative decision making. The strategic partnership means cooperation and coordination of actions through the supply chain. The strategic partnerships change material, financial and information flows among participants in the supply chain. The way of information sharing is changed by information centralizing using information technology. The expected result is mutually beneficial, win-win partnership that creates a synergistic supply chain in which the entire chain is more effective than the sum of its individual parts. Supply chain partnership leads to increased information flows, reduced uncertainty, and a more profitable supply chain.

The general supplier-customer relations in supply chain can be taken as centralized or decentralized [3]. The partnership relations are based on supply contracts. Contracts provide a means for bringing the decentralized solution to the centralized solution. Contracts also facilitate long-term partner ship by delineating mutual concessions that favor the persistence of the relationship, as well as specifying penalties for non-cooperative behavior. The contracts are evaluated by multiple criteria as price, quantity, costs, time and quality. There are different approaches to modeling multi-criteria negotiation processes to reach a consensus among partners. A cooperative decision making requires free communication among users and gives synergic effects in a conflict resolution. The basic trend in the cooperative decision making is to transform a possible conflict to a joint problem. Some basic ideas of formal approaches of problem solving can be introduced to cooperative decision making. There are two aspects of the problem solving - representation and searching. The state space representation introduces the concepts of states and operators. A solution could be obtained by a search process that first applies operators to the initial state to produce new states and so on, until the goal state is produced. Communication between producers and retailers can be provided through information sharing (see Figure. 1). 


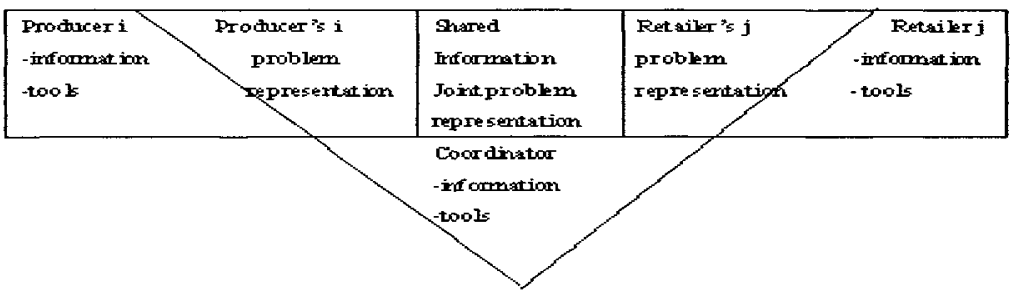

Figure 1. Information Sharing in Supply Chain

The proposed model is a discrete dynamic model and the cooperation of units is based on contracts and formal agreements achieved in negotiation process. We propose a two phases' interactive approach for solving cooperative decision making problems [4]:

1. Finding the ideal solution for individual users.

2. Finding a consensus for all the users.

In the first phase every decision maker searches the ideal alternative. In the second phase a consensus could be obtained by the search process and the principle of cooperativeness is applied.

The negotiation process modeling in general is a complex problem based on several kernel ideas. The framework [3] of the proposed discrete dynamic model is separated to three parts

1. Deterministic part,

2. Logical part,

3. Stochastic part.

\section{MODELING OF SHARING INFORMATION IN INDUSTRY CHAIN}

The layers of among producers compete in a non-cooperative way, but the rivals in the same industry profit from cooperative decision making. The strategic partnership means cooperation and coordination of actions through the Service-oriented architecture. The strategic partnerships change material, financial and information flows among participants in SOA. The way of information sharing is changed by information centralizing using information technology. The partnership leads to increased information flows, reduced uncertainty, and a more profitable industry chain.

The partnership relations are based on cooperation protocols. Protocols provide a means for bringing the decentralized solution to the centralized solution. Protocols also facilitate long-term partner ship by delineating mutual concessions that favor the persistence of the relationship. There are different approaches to modeling multicriteria negotiation processes to reach a consensus among partners. A cooperative decision making requires free communication among producer and gives synergic 
effects in a conflict resolution. The basic trend in the cooperative decision making is to transform a possible conflict to a joint problem. Some basic ideas of formal approaches of problem solving can be introduced to cooperative decision making. There are two aspects of the problem solving - representation and searching. The state space representation introduces the concepts of states and operators. A solution could be obtained by a search process that first applies operators to the initial state to produce new states and so on, until the goal state is produced. Communication between producers can be provided through information sharing (see Figure2).

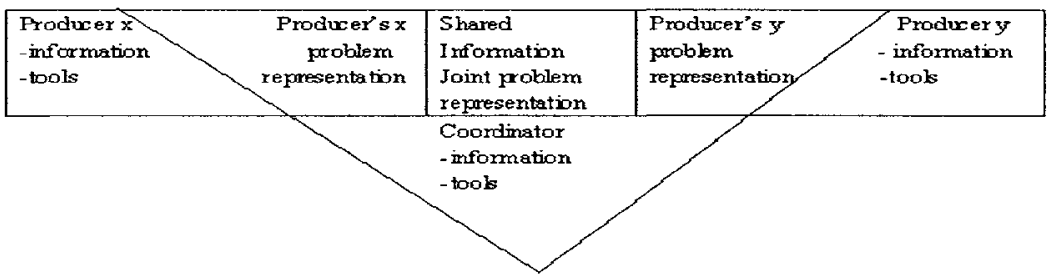

Figure 2. Information Sharing in Industry Chain

The proposed model is a discrete dynamic model and the cooperation of units is based on protocols and formal agreements achieved in negotiation process. We propose a two phases' interactive approach for solving cooperative decision making problems:

1. Finding the ideal solution for industry chains.

2. Finding a consensus for all the chains.

In the first phase every decision maker searches the ideal alternative. In the second phase a consensus could be obtained by the search process and the principle of cooperativeness is applied. The heuristic information for the decision making unit is the distance between this proposal and the opponent's proposal.

\section{A MODEL OF SHARING SERVICE}

From Figure land Figure2, we can design a generic model of sharing services for the same users or different users in the same chain. Because Service Oriented Architecture is currently the most promising architecture for building services; we propose to use SOA in the construction of sharing services. We start with the modeling of a generic sharing service and then map it to a SOA environment. A SOA framework will then be elaborated based on the findings identified from the mapping. Fig. 3 depicts the logical architecture of a sharing service that is used by three users.

Following the model of a generic service proposed in [5], a sharing service can be represented by four components: Service Logic, Service Data, Service Content and Service Profile.

Service Logic is the program code that constitutes the dynamic behavior and provides the functions of a service. The logic of a mobile service can be subject to various distributions, as in any distributed system [6]. Service Data are used in the execution of the service logic and reflecting the state of it. They are for example 
variable values, temporal parameters, register values, stack values, counting parameters, etc.

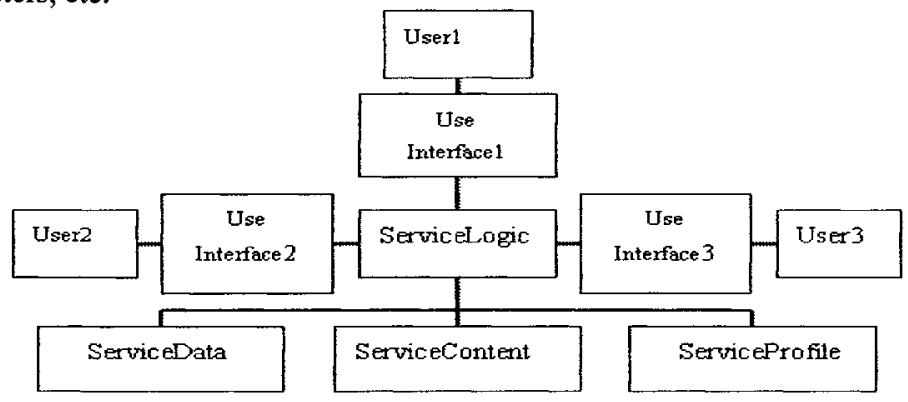

Figure 3. Logical Architecture of a Sharing Service

Service Content refers to data that are the product of service usage. For example it can be a document written in a word processor or the entries in a calendar. Service content can be produced or consumed by the user. Service Profile contains the settings that are related to the user or/and the accessing device.

Each user employs a User Interface to collaborate with the other users via the Collaboration Service. The User Interface can be a generic component that can be used to access several services like a browser. It could be a dedicated component that is especially built for a specific service. Each user can use different instances of the same implementation, e.g. different instances of Internet Explorer. These components can be referred to as identical components. They can also use different instances of different implementations. These components can be referred to as equivalent components.

\section{MODEL REFINEMENTS}

In the context of the simple SCM defined in Section 3 , the usage of our modeling framework is illuminated in Section 4, Section 5 and Section 6 . Since the services are elicited from business requirements, their related service components can be specified. Then, for architectural reuse and interoperability, the system architecture should be defined. The architecture specification should be given. Hereinto, it involves service component searching problem. And our before work on SE4SC [7] can support the query of matched service components in the repository.

With respect of the high dynamic nature, dynamic configuration and reconfiguration of SOA according to the changing requirements and environments at runtime and evolving as execution processes are supported here. SOA allows its services consumer to replace services at run-time when there are some new services, which is a better alternative to the former one concerning functionality or quality, or if a service is not reachable any longer because of network problems. And our method can support the dynamic configuration and reconfiguration of SOA by remove or modifying the usage dependency between different service components. Besides, graph transformation rules referred in [8] may be helpful, too. 
Then, we can refine the architecture specification defined in figure 4. We suppose that there are an alternative retailer service component and an alternative manufacturer service component in the SCM. On account of that the instance of the retailer service component are running on the unsteady thread in the Internet, it may collapse or stop without notification. In these cases, in order to satisfy the needs of customer all the time, it may require the instance to restart or request a substitute. Otherwise, when the published services provided by a service component have higher quality and reliability, consumer will be notified and it may rebind the new service component. According to above discussion, the refined architecture in the SCM is defined in Figure4.

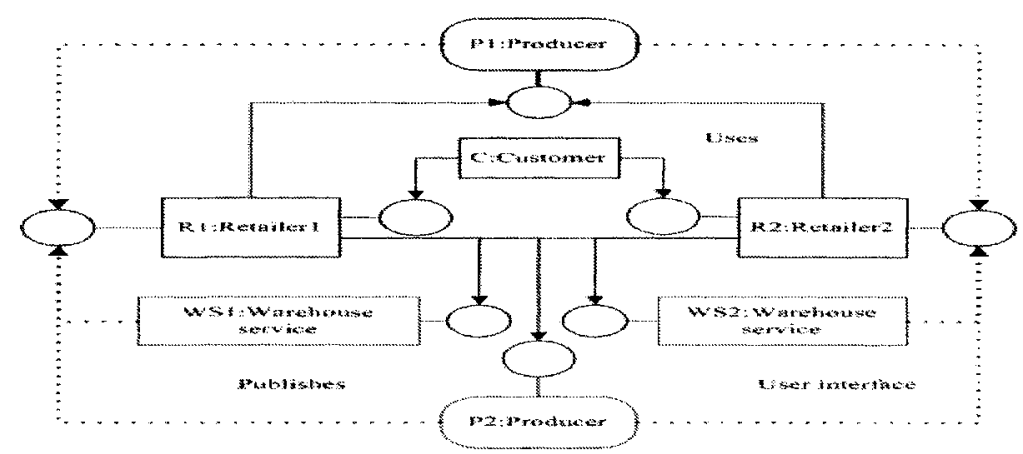

Figure 4. Refined Architecture in the SCM

\section{CONCLUSION}

SCM has generated a substantial amount of interest both among managers and researchers. The interest has also been fuelled by the growth in the development and application of e-business technologies. E-business is associated with business models and practices enabling continuous improvements in supply chains. SCM is more and more affected by network and dynamic business environment and by information and communication technologies.

In this paper the Service Oriented Architecture is investigated and found feasible for the construction of sharing services. The generic model of sharing service is mapped to SOA. To alleviate the tasks of the producers, the basic sharing services can be built by composing or by orchestrating the sharing services together with other services.

The paper is devoted to modeling of supply chain. In the model some important features of this environment are established. The combination of network structure modeling in supply chain can be a powerful instrument of performance analysis of supply chains.

The proposed modeling framework is composed from three inter-related network structures: a production net. The production net captures information flows among 
agents. SOA modeling is important to the service oriented system development, this paper describes problem of SOA within the context of the SCM and proposes a modeling framework as solution.

The paper highlights the describing problem of SOA within the context of the SCM and proposes a modeling framework as solution, it help enterprises in China mainland to resolve their SCM with numbered money: from modeling of sharing information in industry chain and in supply chains, modeling of sharing services, to model refinement; all these models improve enterprises in China mainland competitive power, make them can catch up with their international rivals.

\section{REFERENCES}

1. A.M. Eric, Build a Better Enterprise Application, Network Magazine. Volume 19, Number 8, pp.18-24, (2004).

2. W3C, W3C Working Group Note 11: Web Services Architecture, World Wide Web Consortium, eds. D. Booth, M. Champion, C. Ferris, F. McCabe, E. Newcomer, and D. Orchard (2004). http://www.w3.org/TR/ws-arch/\#stakeholder (Accessed December 2, 2006).

3. P. Fiala, Modeling of relations in supply chains, The Joumal of Business Perspective. Special Issue on Supply Chain Management, pp.127-131, (2003).

4. P. Fiala, Models of cooperative decision making, in multiple criteria decision making, eds. T. Gal and G. Fandel (Springer: Berlin, 1997).

5. I. Jorstad, D. Thanh, and S. Dustdar, An analysis of service continuity in mobile services, in Proc. of the 2nd International Workshop on Distributed and Mobile Collaboration (IEEE Computer Society Press: Los Alamitos, CA, USA, 2004), pp.121-126.

6. ISO. Open Distributed Processing Reference Model - parts 1, 2, 3, 4. ISO 10746-1, 2, 3, 4 (OMG: Needham, MA, USA, 1995).

7. H. Chen, S. Ying, J. Liu, and W. Wang, SE4SC: a Specific Search Engine for Software Components, in Proc. 4th International Conf. on Computer and Information Technology (Wu Han, China, 2004), pp.863-868.

8. L. Baresi, R. Heckel, S. Thöne, and D. Varró, Modeling and Analysis of Architectural Styles based on Graph Transformation, in Proc. 25th Int. Conf. Sofiware Engineering Workshop on CBSE6: Automated Reasoning and Prediction (Portland, Oregon, USA, 2003), pp.67-72. 